\title{
QUASI-ISOMETRIC CO-HOPFICITY OF NON-UNIFORM LATTICES IN RANK-ONE SEMI-SIMPLE LIE GROUPS
}

\author{
ILYA KAPOVICH AND ANTON LUKYANENKO
}

\begin{abstract}
We prove that if $G$ is a non-uniform lattice in a rank-one semisimple Lie group $\neq \operatorname{Isom}\left(\mathbb{H}_{\mathbb{R}}^{2}\right)$, then $G$ is quasi-isometrically co-Hopf. This means that every quasi-isometric embedding $G \rightarrow G$ is coarsely surjective and thus is a quasi-isometry.
\end{abstract}

\section{INTRODUCTION}

The notion of co-Hopficity plays an important role in group theory. Recall that a group $G$ is said to be co-Hopf if $G$ is not isomorphic to a proper subgroup of itself, that is, if every injective homomorphism $G \rightarrow G$ is surjective. A group $G$ is almost co-Hopf if for every injective homomorphism $\phi: G \rightarrow G$ we have $[G: \phi(G)]<\infty$. Clearly, being co-Hopf implies being almost co-Hopf. The converse is not true: for example, for any $n \geq 1$, the free abelian group $\mathbb{Z}^{n}$ is almost co-Hopf but not co-Hopf.

It is easy to see that any freely decomposable group is not co-Hopf. In particular, a free group of rank at least 2 is not co-Hopf. It is also well known that finitely generated nilpotent groups are always almost co-Hopf and, under some additional restrictions, also co-Hopf [1. An important result of Sela [17. states that a torsion-free non-elementary word-hyperbolic group $G$ is co-Hopf if and only if $G$ is freely indecomposable. Partial generalizations of this result are known for certain classes of relatively hyperbolic groups, by the work of Belegradek and Szczepański [2]. Co-Hopficity has also been extensively studied for 3-manifold groups and for Kleinian groups. Delzant and Potyagailo 9] gave a complete characterization of co-Hopfian groups among non-elementary geometrically finite Kleinian groups without 2-torsion.

A counterpart algebraic notion is that of Hopficity. A group $G$ is said to be Hopfian if every surjective endomorphism $G \rightarrow G$ is necessarily injective, and hence is an automorphism of $G$. This notion is also extensively studied in geometric group theory. In particular, an important result of Sela [18] shows that every torsion-free word-hyperbolic group is Hopfian. The notion of Hopficity admits a number of interesting "virtual" variations. Thus a group $G$ is called cofinitely Hopfian if every endomorphism of $G$ whose image is of finite index in $G$, is an automorphism of $G$; see, for example [7.

Received by the editors April 17, 2012.

2010 Mathematics Subject Classification. Primary 20F65, Secondary 53C23.

The first author was supported by the NSF grant DMS-0904200.

The authors acknowledge support from the National Science Foundation grant DMS-1107452 "RNMS: Geometric structures and representation varieties". 
A key general theme in geometric group theory is the study of "large-scale" geometric properties of finitely generated groups. Recall that if $\left(X, d_{X}\right)$ and $\left(Y, d_{Y}\right)$ are metric spaces, a map $f: X \rightarrow Y$ is called a coarse embedding if there exist monotone non-decreasing functions $\alpha, \omega:[0, \infty) \rightarrow \mathbb{R}$ such that $\alpha(t) \leq \omega(t)$, that $\lim _{t \rightarrow \infty} \alpha(t)=\infty$, and such that for all $x, x^{\prime} \in X$ we have

$$
\alpha\left(d_{X}\left(x, x^{\prime}\right)\right) \leq d_{Y}\left(f(x), f\left(x^{\prime}\right)\right) \leq \omega\left(d_{X}\left(x, x^{\prime}\right)\right) .
$$

If $d_{X}$ is a path metric, then for any coarse embedding $f: X \rightarrow Y$ the function $\omega(t)$ can be chosen to be affine, that is, of the form $\omega(t)=a t+b$ for some $a, b \geq 0$.

A coarse map $f$ is called a coarse equivalence if $f$ is coarsely surjective, that is, if there is $C \geq 0$ such that for every $y \in Y$ there exists $x \in X$ with $d_{Y}(y, f(x)) \leq C$. A map $f: X \rightarrow Y$ is called a quasi-isometric embedding if $f$ is a coarse embedding and the functions $\alpha(t), \omega(t)$ in $(*)$ can be chosen to be affine, that is, of the form $\alpha(t)=\frac{1}{\lambda} t-\epsilon, \omega(t)=\lambda t+\epsilon$ where $\lambda \geq 1, \epsilon \geq 0$. Finally, a map $f: X \rightarrow Y$ is a quasi-isometry if $f$ is a quasi-isometric embedding and $f$ is coarsely surjective.

The notion of co-Hopficity has the following natural counterpart for metric spaces. We say that a metric space $X$ is quasi-isometrically co-Hopf if every quasiisometric embedding $X \rightarrow X$ is coarsely surjective, that is, if every quasi-isometric embedding $X \rightarrow X$ is a quasi-isometry. More generally, a metric space $X$ is called coarsely co-Hopf if every coarse embedding $X \rightarrow X$ is coarsely surjective. Clearly, if $X$ is coarsely co-Hopf, then $X$ is quasi-isometrically co-Hopf. If $G$ is a finitely generated group with a word metric $d_{G}$ corresponding to some finite generating set of $G$, then every injective homomorphism $G \rightarrow G$ is a coarse embedding. This easily implies that if $\left(G, d_{G}\right)$ is coarsely co-Hopf, then the group $G$ is almost co-Hopf.

Example 1.1. The real line $\mathbb{R}$ is coarsely co-Hopf (and hence quasi-isometrically co-Hopf). This follows from the fact that any coarse embedding must send the ends of $\mathbb{R}$ to distinct ends. Since $\mathbb{R}$ has two ends, a coarse embedding induces a bijection on the set of ends of $\mathbb{R}$. It is then not hard to see that a coarse embedding from $\mathbb{R}$ to $\mathbb{R}$ must be coarsely surjective. See [6] for the formal definition of ends of a metric space.

Example 1.2. The rooted regular binary tree $T_{2}$ is not quasi-isometrically co-Hopf. We can identify the set of vertices of $T_{2}$ with the set of all finite binary sequences. The root of $T_{2}$ is the empty binary sequence $\epsilon$ and for a finite binary sequence $x$ its left child is the sequence $0 x$ and the right child is the sequence $1 x$. Consider the map $f: T_{2} \rightarrow T_{2}$ which maps $T_{2}$ isometrically to a copy of itself that "hangs below" the vertex 0 . Thus $f(x)=0 x$ for every finite binary sequence $x$. Then $f$ is an isometric embedding but the image $f\left(T_{2}\right)$ is not co-bounded in $T_{2}$ since it misses the entire infinite branch located below the vertex 1 .

Example 1.3. Consider the free group $F_{2}=F(a, b)$ on two generators. Then $F_{2}$ is not quasi-isometrically co-Hopf.

The Cayley graph $X$ of $F_{2}$ is a regular 4-valent tree with every edge of length 1 . We may view $X$ in the plane so that every vertex has one edge directed upward, and three downward. Picking a vertex $v_{0}$ of $X$, denote its left branch by $X_{1}$ and the remainder of the tree by $X_{2}$. We have $X_{1} \cup X_{2}=X$, and $X_{1}$ is a rooted ternary tree. Define a quasi-isometric embedding $f: X \rightarrow X$ by taking $f$ to be a shift on $X_{1}$ (defined similarly to Example 1.2) and the identity on $X_{2}$. The map $f$ is not coarsely surjective, but it is a quasi-isometric embedding. Moreover, for any vertices $x, x^{\prime}$ of $X$ we have $\left|d\left(f(x), f\left(x^{\prime}\right)\right)-d\left(x, x^{\prime}\right)\right| \leq 1$. 
One can also see that $F_{2}=F(a, b)$ is not quasi-isometrically co-Hopf for algebraic reasons. Let $u, v \in F(a, b)$ with $[u, v] \neq 1$. Then there is an injective homomorphism $h: F(a, b) \rightarrow F(a, b)$ such that $h(a)=u$ and $h(b)=v$. This homomorphism $f$ is always a quasi-isometric embedding of $F(a, b)$ into itself.

If, in addition, $u$ and $v$ are chosen so that $\langle u, v\rangle \neq F(a, b)$, then $[F(a, b)$ : $h(F(a, b))]=\infty$ and the image $h(F(a, b))$ is not co-bounded in $F(a, b)$.

Thus, the group $F_{2}$ is not almost co-Hopf and not quasi-isometrically co-Hopf.

Example 1.4. There do exist finitely generated groups that are algebraically coHopf but not quasi-isometrically co-Hopf. The simplest example of this kind is the solvable Baumslag-Solitar group $B(1,2)=\left\langle a, t \mid t^{-1} a t=a^{2}\right\rangle$. It is well known that $B(1,2)$ is co-Hopf.

To see that $B(1,2)$ is not quasi-isometrically co-Hopf we use the fact that $B(1,2)$ admits an isometric properly discontinuous co-compact action on a proper geodesic metric space $X$ that is "foliated" by copies of the hyperbolic plane $\mathbb{H}_{\mathbb{R}}^{2}$. We refer the reader to the paper of Farb and Mosher 12 for a detailed description of the space $X$, and will only briefly recall the properties of $X$ here.

Topologically, $X$ is homeomorphic to the product $\mathbb{R} \times T_{3}$ where $T_{3}$ is an infinite 3-regular tree (drawn upwards): there is a natural projection $p: X \rightarrow T_{3}$ whose fibers are homeomorphic to $\mathbb{R}$. The boundary of $T_{3}$ is decomposed into two sets: the "lower boundary" consisting of a single point $u$ and the "upper boundary" $\partial_{\delta} X$ which is homeomorphic to the Cantor set (and can be identified with the set of dyadic rationals). For any bi-infinite geodesic $\ell$ in $T_{3}$ from $u$ to a point of $\partial_{\delta} X$ the full-p-preimage of $\ell$ in $X$ is a copy of the hyperbolic plane $\mathbb{H}_{\mathbb{R}}^{2}$ (in the upper-half plane model). The $p$-preimage of any vertex of $T_{3}$ is a horizontal horocycle in the $\mathbb{H}_{\mathbb{R}^{-}}^{2}$ "fibers". Any two $\mathbb{H}^{2}$-fibers intersect along a complement of a horoball in $\mathbb{H}_{\mathbb{R}}^{2}$.

Similar to the above example for $F(a, b)$, we can take a quasi-isometric embedding $f: T_{3} \rightarrow T_{3}$ whose image misses an infinite subtree in $T_{3}$ and such that $\left|d\left(x, x^{\prime}\right)-d\left(f(x), f\left(x^{\prime}\right)\right)\right| \leq 1$ for any vertices $x, x^{\prime}$ of $T_{3}$. It is not hard to see that this map $f$ can be extended along the $p$-fibers to a map $\tilde{f}: X \rightarrow X$ such that $\tilde{f}$ is a quasi-isometric embedding but not coarsely surjective. Since $X$ is quasi-isometric to $B(1,2)$, it follows that $B(1,2)$ is not quasi-isometrically co-Hopf.

Example 1.5. Grigorchuk's group $G$ of intermediate growth provides another intersting example of a group that is not quasi-isometrically co-Hopf. This group $G$ is finitely generated and can be realized as a group of automorphisms of the regular binary rooted tree $T_{2}$. The group $G$ has a number of unusual algebraic properties: it is an infinite 2-torsion group, it has intermediate growth, it is amenable but not elementary amenable and so on. See Chapter VIII in [8] for detailed background on the Grigorchuk group. It is known that there exists a subgroup $K$ of index 16 in $G$ such that $K \times K$ is isomorphic to a subgroup of index 64 in $G$. The map $K \rightarrow K \times K, k \mapsto(k, 1)$ is clearly a quasi-isometric embedding which is not coarsely surjective. Since both $K$ and $K \times K$ are quasi-isometric to $G$, it follows that $G$ is not quasi-isometrically co-Hopf.

For Gromov-hyperbolic groups and spaces, quasi-isometric co-Hopficity is closely related to the properties of their hyperbolic boundaries. We say that a compact metric space $K$ is topologically co-Hopf if $K$ is not homeomorphic to a proper 
subset of itself. We say that $K$ is quasi-symmetrically co-Hopf if every quasisymmetric map $K \rightarrow K$ is surjective. Note that for a compact metric space $K$ being topologically co-Hopf obviously implies being quasi-symmetrically co-Hopf.

Example 1.6. A recent important result of Merenkov [15] shows that the converse implication does not hold. He constructed a round Sierpinski carpet $\mathbb{S}$ such that $\mathbb{S}$ is quasi-symmetrically co-Hopf. Since $\mathbb{S}$ is homeomorphic to the standard "square" Serpinski carpet, clearly $\mathbb{S}$ is not topologically co-Hopf.

It is well known (see, for example, [3]) that if $X, Y$ are proper Gromov-hyperbolic geodesic metric spaces, then any quasi-isometric embedding $f: X \rightarrow Y$ induces a quasi-symmetric topological embedding $\partial f: \partial X \rightarrow \partial Y$ between their hyperbolic boundaries. It is then not hard to see that if $G$ is a word-hyperbolic group whose hyperbolic boundary $\partial G$ is quasi-symmetrically co-Hopf (e.g. if it is topologically co-Hopf), then $G$ is quasi-isometrically co-Hopf. This applies, for example, to any word-hyperbolic groups whose boundary $\partial G$ is homeomorphic to an $n$-sphere (with $n \geq 1$ ), such as fundamental groups of closed Riemannian manifolds with all sectional curvatures $\leq-1$.

The main result of this paper is the following:

Theorem 1.7. Let $G$ be a non-uniform lattice in a rank-one semi-simple real Lie group other than $\operatorname{Isom}\left(\mathbb{H}_{\mathbb{R}}^{2}\right)$. Then $G$ is quasi-isometrically co-Hopf.

Thus, for example, if $M$ is a complete finite volume non-compact hyperbolic manifold of dimension $n \geq 3$, then $\pi_{1}(M)$ is quasi-isometrically co-Hopf. Note that if $G$ is a non-uniform lattice in $\operatorname{Isom}\left(\mathbb{H}_{\mathbb{R}}^{2}\right)$, then the conclusion of Theorem 1.7 does not hold since $G$ is a virtually free group.

If $G$ is a uniform lattice in a rank-one semi-simple real Lie group (including possibly a lattice in $\operatorname{Isom}\left(\mathbb{H}_{\mathbb{R}}^{2}\right)$ ), then $G$ is Gromov-hyperbolic with the boundary $\partial G$ being homeomorphic to $\mathbb{S}^{n}$ (for some $n \geq 1$ ). In this case it is easy to see that $G$ is also quasi-isometrically co-Hopf since every topological embedding from $\mathbb{S}^{n}$ to itself is necessarily surjective.

Convention 1.8. From now on and for the remainder of this paper let $X \neq \mathbb{H}_{\mathbb{R}}^{2}$ be a rank-one negatively curved symmetric space with metric $d_{X}$ (or just d in most cases). Namely, $X$ is isometric to a hyperbolic space $\mathbb{H}_{\mathbb{R}}^{n}$ (with $n \geq 3$ ), $\mathbb{H}_{\mathbb{C}}^{n}$ (with $n \geq 2), \mathbb{H}_{H}^{n}$ over the reals, complexes, or quaternions, or to the octonionic plane $\mathbb{H}_{\mathbb{O}}^{2}$.

If $G$ is as in Theorem 1.7, then $G$ acts properly discontinuously (but with a non-compact quotient) by isometries on such a space $X$ and there exists a $G$ invariant collection $\mathcal{B}$ of disjoint horoballs in $X$ such that $(X \backslash \mathcal{B}) / G$ is compact. The "truncated" space $\Omega=X \backslash \mathcal{B}$, endowed with the induced path-metric $d_{\Omega}$ is quasi-isometric to the group $G$ by the Milnor-Schwartz Lemma. Thus it suffices to prove that $\left(\Omega, d_{\Omega}\right)$ is quasi-isometrically co-Hopf.

Richard Schwartz [16] established quasi-isometric rigidity for non-uniform lattices in rank-one semi-simple Lie groups and we use his proof as a starting point.

First, using coarse cohomological methods (particularly techniques of KapovichKleiner [14]), we prove that spaces homeomorphic to $\mathbb{R}^{n}$ with "reasonably nice" metrics are coarsely co-Hopf. This result applies to the Euclidean space $\mathbb{R}^{n}$ itself, to simply connected nilpotent Lie groups, to the rank-one symmetric spaces $X$ mentioned above, as well as to the horospheres in $X$. Let $f:\left(\Omega, d_{\Omega}\right) \rightarrow\left(\Omega, d_{\Omega}\right)$ 
be a quasi-isometric embedding. Schwartz' work implies that for every peripheral horosphere $\sigma$ in $\Omega$ there exists a unique peripheral horosphere $\sigma^{\prime}$ of $X$ such that $f(\sigma)$ is contained in a bounded neighborhood of $\sigma^{\prime}$. Using coarse co-Hopficity of horospheres, mentioned above, we conclude that $f$ gives a quasi-isometry (with controlled constants) between $\sigma$ and $\sigma^{\prime}$. Then, following Schwartz, we extend the map $f$ through each peripheral horosphere to the corresponding peripheral horoball $B$ in $X$. We then argue that the extended map $\hat{f}: X \rightarrow X$ is a coarse embedding. Using coarse co-Hopficity of $X$, it follows that $\hat{f}$ is coarsely surjective, which implies that the original map $f:\left(\Omega, d_{\Omega}\right) \rightarrow\left(\Omega, d_{\Omega}\right)$ is coarsely surjective as well.

It seems likely that the proof of Theorem 1.7 generalizes to an appropriate subclass of relatively hyperbolic groups. However, a more intriguing question is to understand what happens for higher-rank lattices.

Problem 1.9. Let $G$ be a non-uniform lattice in a semi-simple real Lie group of rank $\geq 2$. Is $G$ quasi-isometrically co-Hopf?

Unlike the groups considered in the present paper, higher-rank lattices are not relatively hyperbolic. Quasi-isometric rigidity for higher-rank lattices is known to hold, by the result of Eskin [11, but the proofs there are quite different from the proof of Schwartz in the rank-one case.

Another natural question is:

Problem 1.10. Let $G$ be as in Theorem 1.7 Is $G$ coarsely co-Hopf?

Our proof only yields quasi-isometric co-Hopficity, and it is possible that coarse co-Hopficity actually fails in this context.

The result of Merenkov (Example 1.6) produces the first example of a compact metric space $K$ which is quasi-symmetrically co-Hopf but not topologically coHopf. Topologically, $K$ is homeomorphic to the standard Sierpinkski carpet and there exists a word-hyperbolic group (in fact, a Kleinian group) with boundary homeomorphic to $K$. However, the metric structure on the Sierpinski carpet in Merenkov's example is not "group-like" and is not quasi-symmetric to the visual metric on the boundary of a word-hyperbolic group.

Problem 1.11. Does there exist a word-hyperbolic group $G$ such that $\partial G$ (with the visual metric) is quasi-symmetrically co-Hopf (and hence $G$ is quasi-isometrically co-Hopf), but such that $\partial G$ is not topologically co-Hopf? In particuar, do there exist examples of this kind where $\partial G$ is homeomorphic to the Sierpinski carpet or the Menger curve?

The above question is particularly interesting for the family of hyperbolic buildings $I_{p, q}$ constructed by Bourdon and Pajot [5, 4. In their examples $\partial I_{p, q}$ is homeomorphic to the Menger curve, and it turns out to be possible to precisely compute the conformal dimension of $\partial I_{p, q}$. Note that, similar to the Sierpinski carpet, the Menger curve is not topologically co-Hopf.

Problem 1.12. Are the Burdon-Pajot buildings $I_{p, q}$ quasi-isometrically co-Hopf? Equivalently, are their boundaries $\partial I_{p, q}$ quasi-symmetrically co-Hopf?

It is also interesting to investigate quasi-isometric and coarse co-Hopficity for other natural classes of groups and metric spaces. In an ongoing work (in preparation), Jason Behrstock, Alessandro Sisto, and Harold Sultan study quasi-isometric co-Hopficity for mapping class groups and also characterize exactly when this property holds for fundamental groups of 3-manifolds. 


\section{Geometric objects}

2.1. Horoballs. Recall that, by Convention 1.8, $X$ is a rank one symmetric space different from $\mathbb{H}_{\mathbb{R}}^{2}$. Namely, $X$ is isometric to a hyperbolic space $\mathbb{H}_{\mathbb{R}}^{n}$ (with $n \geq 3$ ), $\mathbb{H}_{\mathbb{C}}^{n}(n \geq 2), \mathbb{H}_{H}^{n}$ over the reals, complexes, or quaternions, or to the octonionic plane $\mathbb{H}_{\mathbb{O}}^{2}$. We recall some properties of $X$; see [6], Chapter II.10, for details.

Definition 2.1. Let $0 \in X$ be a basepoint and $\gamma$ a geodesic ray starting at 0 . The associated function $b: X \rightarrow \mathbb{R}$ given by

$$
b(x)=\lim _{s \rightarrow \infty} d(x, \gamma(s))-s
$$

is known as a Busemann function on $X$. A horosphere is a level set of a Busemann function. The set $b^{-1}\left[t_{0}, \infty\right) \subset X$ is a horoball. Up to the action of the isometry group on $X$, there is a unique Busemann function, horosphere, and horoball.

A Busemann function $b(x)$ provides a decomposition of $X$ into horospherical coordinates, a generalization of the upper-halfspace model. Namely, let $\sigma=b^{-1}(0)$ and decompose $X=\sigma \times \mathbb{R}^{+}$as follows: given $x \in X$, flow along the gradient of $b$ for time $b(x)$ to reach a point $s \in \sigma$, and write $x=\left(s, e^{b(x)}\right)$. In horospherical coordinates, the $\sigma$-fibers $\{s\} \times \mathbb{R}^{+}$are geodesics, the $\mathbb{R}^{+}$-fibers $\sigma \times\left\{t_{0}\right\}$ are horospheres, and the sets $\sigma \times\left[t_{0}, \infty\right)$ are horoballs. Other horoballs appear as closed balls tangent to the boundary $\sigma \times\{0\}$.

If $(M, d)$ is a metric space and $C \geq 0$, a path $\gamma:[a, b] \rightarrow M$, parameterized by arc-length, is called a $C$-rough geodesic in $M$, if for any $t_{1}, t_{2} \in[a, b]$ we have

$$
\left|d\left(\gamma\left(t_{1}\right), \gamma\left(t_{2}\right)\right)-\right| t_{1}-t_{2}|| \leq C .
$$

If $Y, Y^{\prime}$ are metric spaces, a map $f: Y \rightarrow Y^{\prime}$ is coarsely Lipschitz if there exists $C>0$ such that for any $y_{1}, y_{2} \in Y$ we have $d_{Y^{\prime}}\left(f\left(y_{1}\right), f\left(y_{2}\right)\right) \leq C d_{Y}\left(y_{1}, y_{2}\right)$. If $Y$ is a path metric space, then it is easy to see that $f: Y \rightarrow Y^{\prime}$ is coarsely Lipschitz if and only if there exist constants $C, C^{\prime}>0$ such that for any $y_{1}, y_{2} \in Y$ with $d_{Y}\left(y_{1}, y_{2}\right) \leq C$ we have $d_{Y^{\prime}}\left(f(y), f\left(y^{\prime}\right)\right) \leq C^{\prime}$.

The following two lemmas appear to be well-known folklore facts:

Lemma 2.2. There exists $C>0$ with the following property: Let $\mathcal{B}$ be a horoball in $X, x_{1} \in X \backslash \mathcal{B}$ and $x_{2} \in \mathcal{B}$. Let $b$ be the point in $\mathcal{B}$ closest to $x_{1}$. Then the piecewise geodesic $\left[x_{1}, b\right] \cup\left[b, x_{2}\right]$ is a $C$-rough geodesic.

Proof. Acting by isometries of $X$, we may assume that $\mathcal{B}$ is a fixed horoball that is tangent to the boundary of $X$ in the horospherical model. We may also assume that $b$ is the top-most point of $\mathcal{B}$, so that $x_{1}$ lies in the vertical geodesic passing through $b$; see Figure 1 .

Consider the "top" of $\mathcal{B}$, i.e. the maximal subset of $\partial \mathcal{B}$ that is a graph in horospherical coordinates. Considering the Riemannian metric on $X$ in horospherical coordinates, one sees that the geodesic $\left[x_{1}, x_{2}\right]$ must pass through the top of $\mathcal{B}$. Setting $C$ to be the radius of the top of $\mathcal{B}$, centered at $b$, completes the proof.

Lemma 2.3. Let $\mathcal{B}_{1}, \mathcal{B}_{2}$ be disjoint horoballs, and $x_{1} \in \mathcal{B}_{1}, x_{2} \in \mathcal{B}_{2}$. Let $\left[b_{1}, b_{2}\right]$ be the minimal geodesic between $\mathcal{B}_{1}$ and $\mathcal{B}_{2}$. Then $\left[x_{1}, b_{1}\right] \cup\left[b_{1}, b_{2}\right] \cup\left[b_{2}, x_{2}\right]$ is a $C$-rough geodesic, for the value of $C$ in Lemma 2.2 .

Proof. The proof is analagous to that of Lemma 2.2. We may normalize the horoballs $\mathcal{B}_{1}, \mathcal{B}_{2}$ as in Figure 2. The normalization depends only on the distance 


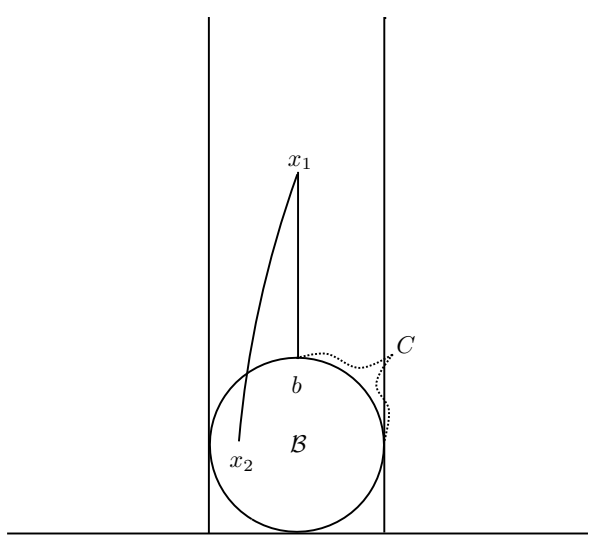

FiguRE 1. Lemma 2.2 for $X=\mathbb{H}_{\mathbb{R}}^{2}$.

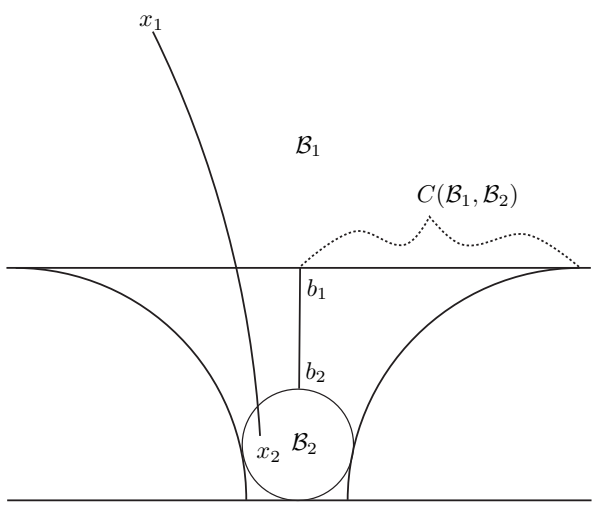

Figure 2. Lemma 2.3 for $X=\mathbb{H}_{\mathbb{R}}^{2}$.

$d\left(\mathcal{B}_{1}, \mathcal{B}_{2}\right)$. Any geodesic $\left[x_{1}, x_{2}\right]$ must then pass through compact regions near $b_{1}$ and $b_{2}$. Let $C\left(\mathcal{B}_{1}, \mathcal{B}_{2}\right)$ be the radius of this region in $\mathcal{B}_{1}$. Fixing $\mathcal{B}_{1}$ and varying $\mathcal{B}_{2}$, set $C=\sup C\left(\mathcal{B}_{1}, \mathcal{B}_{2}\right)$. The value $C\left(\mathcal{B}_{1}, \mathcal{B}_{2}\right)$ remains bounded if the distance between the horoballs goes to infinity (converging to the constant $C$ in Lemma 2.2). Thus, the infimum is attained and $C<\infty$. This completes the proof.

Lemma 2.4. Let $\mathcal{B}_{1}, \mathcal{B}_{2}$ be disjoint horoballs, $x_{1} \in \mathcal{B}_{1}, x_{2} \in \mathcal{B}_{2}$. Denote the minimal geodesic between $\mathcal{B}_{1}$ and $\mathcal{B}_{2}$ by $\left[b_{1}, b_{2}\right]$. Then $d\left(x_{1}, b_{1}\right) \leq d\left(x_{1}, x_{2}\right)$.

Proof. Fix $D>0$ and allow $\mathcal{B}_{1}, \mathcal{B}_{2}, x_{1} \in \mathcal{B}_{1}$, and $x_{2} \in \mathcal{B}_{2}$ to vary with the restriction $d\left(x_{1}, x_{2}\right)=D$. Define a function $f$ on the interval $[0, D]$ by

$$
f(t)=\sup \left\{d\left(x_{1}, b_{1}\right): d\left(\mathcal{B}_{1}, \mathcal{B}_{2}\right)=t\right\},
$$

where the supremum is over all combinations of the variables with the restriction stated above, and $b_{1}$ denotes the closest point of $\mathcal{B}_{1}$ to $\mathcal{B}_{2}$. Then $f$ is a decreasing function, since increasing $t$ pushes the horoballs farther apart and forces $x_{1}$ closer to $x_{2}$. In particular, $f(D)=0$ since necessarily $x_{1}=b_{1}$. Conversely, $f(0)=D$, taking $x_{2}=b_{1}=b_{2}$. We then have, for any choice of disjoint $\mathcal{B}_{1}, \mathcal{B}_{2}$ and $x_{1}, x_{2}$ in the corresponding horoballs, that

$$
d\left(x_{1}, b_{1}\right) \leq f\left(d\left(\mathcal{B}_{1}, \mathcal{B}_{2}\right)\right) \leq d\left(x_{1}, x_{2}\right)=D,
$$

as desired.

\subsection{Truncated spaces.}

Definition 2.5. Let $X \neq \mathbb{H}_{\mathbb{R}}^{2}$ be a negatively curved rank one symmetric space. A truncated space $\Omega$ is the complement in $X$ of a set of disjoint open horoballs. A truncated space is equivariant if there is a (non-uniform) lattice $\Gamma \subset \operatorname{Isom}(X)$ that leaves $\Omega$ invariant, with $\Omega / \Gamma$ compact.

We will consider $\Omega$ with the induced path metric $d_{\Omega}$ from $X$. Under this metric, curvature remains negative in the interior of $\Omega$. The curvature on the boundary need not be negative. For an extensive treatment of truncated spaces, see [16]. 
Remark 2.6. Note that truncated spaces are, in general, not uniquely geodesic. Specifically, if $X$ is not a real hyperbolic space, then components of $\partial \Omega$ (which come from horospheres in $X)$ are isometrically embedded in $\left(\Omega, d_{\Omega}\right)$ copies of nonuniquely-geodesic Riemannian metrics on certain nilpotent groups. In particular, $\left(\Omega, d_{\Omega}\right)$ is not necessarily a $C A T(0)$-space.

Remark 2.7. Let $X$ be a negatively curved rank one symmetric space and $\Gamma \subset$ Isom $(X)$ a non-uniform lattice. Then $X / \Gamma$ is a finite-volume manifold with cusps. In $X$, each cusp corresponds to a $\Gamma$-invariant family of horoballs. Removing the horoballs produces an equivariant truncated space $\Omega$ whose quotient $\Omega / \Gamma$ is the compact core of $X / \Gamma$.

Proposition 2.8. Let $X$ be a negatively curved rank one symmetric space and $\Omega \subset X$ an equivariant truncated space. Then the inclusion $\iota:\left(\Omega, d_{\Omega}\right) \hookrightarrow\left(X, d_{X}\right)$ is a coarse embedding.

Proof. Since $d_{\Omega}$ and $d_{X}$ are path metrics with the same line element, we have

$$
d_{X}(x, y) \leq d_{\Omega}(x, y) .
$$

To get the lower bound, define an auxilliary function

$$
\beta(s)=\max \left\{d_{\Omega}(x, y): x, y \in \Omega \text { and } d_{X}(x, y) \leq s\right\} .
$$

Let $K$ be a compact fundamental region for the action of $\Gamma$ on $\Omega$. Because $\Gamma$ acts on $\Omega$ by isometries with respect to both metrics $d_{X}$ and $d_{\Omega}$, we may equivalently define $\beta(s)$ by

$$
\beta(s)=\max \left\{d_{\Omega}(x, y): x \in K, y \in \Omega \text { and } d_{X}(x, y) \leq s\right\} .
$$

Because $K$ is compact and the metrics $d_{X}, d_{\Omega}$ are complete, $\beta(s) \in(0, \infty)$ for $s \in(0, \infty)$. Furthermore, $\beta:[0, \infty] \rightarrow[0, \infty]$ is continuous and increasing, with $\beta(0)=0$. Because horospheres have infinite diameter for both $d_{X}$ and $d_{\Omega}$ (they are isometric to appropriate nilpotent Lie groups with left-invariant Riemannian metrics, see [16]), we also have $\beta(\infty)=\infty$.

Let $\beta^{\prime}$ be an increasing homeomorphism of $[0, \infty]$ with $\beta^{\prime}(s) \geq \beta(s)$ for all $s$ and consider its inverse $\alpha(t)$. For $x, y \in \Omega$ we then have

$$
\begin{aligned}
& d_{\Omega}(x, y) \leq \beta\left(d_{X}(x, y)\right) \leq \beta^{\prime}\left(d_{X}(x, y)\right), \\
& \alpha\left(d_{\Omega}(x, y)\right) \leq d_{X}(x, y) .
\end{aligned}
$$

This concludes the proof.

Remark 2.9. A more precise quantitative version of Proposition 2.8 can be obtained by studying geodesics in $\Omega$; see [10].

2.3. Mappings between truncated spaces. For this section, let $\Omega \subset X$ be a truncated space, with $X \neq \mathbb{H}_{\mathbb{R}}^{2}$, and $f: \Omega \rightarrow \Omega$ a $d_{\Omega}$-quasi-isometric embedding. To ease the exposition, we refer to the target truncated space as $\Omega^{\prime} \subset X^{\prime}$.

Lemma 2.10 (Schwartz [16]). There exists $C>0$ so that for every boundary horosphere $\sigma$ of $\Omega$, there exists a boundary horosphere $\sigma^{\prime}$ of $\Omega^{\prime}$ such that $f(\sigma)$ is contained in a $C$-neighborhood of $\sigma^{\prime}$.

Using nearest-point projection, we may assume $f(\sigma) \subset \sigma^{\prime}$. 
Definition 2.11. Let $\mathcal{B}, \mathcal{B}^{\prime}$ be horoballs with boundaries $\sigma, \sigma^{\prime}$. A point in $\sigma$ corresponds, in horospherical coordinates, to a geodesic ray in $B$. A map $\sigma \rightarrow \sigma^{\prime}$ then extends to a map $\mathcal{B} \rightarrow \mathcal{B}^{\prime}$ in the obvious fashion.

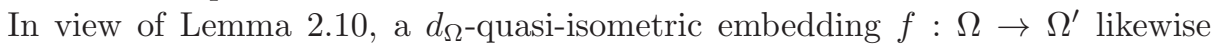
extends to a map $f: X \rightarrow X^{\prime}$ by filling the map on each boundary horoball.

Lemma 2.12 (Schwartz [16]). A quasi-isometry $f: \sigma \rightarrow \sigma^{\prime}$ induces a quasiisometry $\mathcal{B} \rightarrow \mathcal{B}^{\prime}$, with uniform control on constants.

Idea of proof. One considers the metric on the horospheres of $\mathcal{B}$ parallel to $\sigma$, or alternately fixes a model horosphere and varies the metric. One then shows that if $f$ is a quasi-isometry with respect to one of the horospheres, it is also a quasi-isometry with respect to the horospheres at other horo-heights. One then decomposes the metric on $\mathcal{B}$ into a sum of the horosphere metric and the standard metric on $\mathbb{R}$, in horospherical coordinates. This replacement is coarsely Lipschitz, so the extended map is also coarsely Lipschitz. Taking the inverse of $f$ completes the proof.

\section{Compactly supported cohomology}

Definition 3.1. Let $X$ be a simplicial complex and $K_{i} \subset X$ nested compacts with $\bigcup_{i} K_{i}=X$. Compactly supported cohomology $H_{c}^{*}(X)$ is defined by

$$
H_{c}^{*}(X)=\lim _{\longrightarrow} H^{*}\left(X, X \backslash K_{i}\right) .
$$

For a compact space $X, H_{C}^{*}(X)=H^{*}(X)$, but the two do not generally agree for unbounded spaces. We have $H_{c}^{n}\left(\mathbb{R}^{n}\right)=\mathbb{Z}$ and $H_{c}^{n}(\bar{\Omega})=0$ for a non-trivial truncated space $\Omega$. In fact, one has the following lemma.

Lemma 3.2. Let $Z \subset \mathbb{R}^{n}$ be a closed subset. Then $H_{c}^{n}(Z) \neq 0$ if and only if $Z=\mathbb{R}^{n}$.

Proof. It is well known that the choice of nested compact sets does not affect $H_{c}^{n}(Z)$. Choose the sequence $K_{i}=\overline{B(0, i)} \cap Z$, the intersection of a closed ball and $Z$. With respect to the subset topology of $Z$, the boundary of $K_{i}$ is given by $\partial_{Z} K_{i}:=\partial K_{i} \cap \partial \overline{B(0, i)}$. We have by excision

$$
H^{n}\left(Z, K_{i}\right)=H^{n}\left(K_{i}, \partial_{Z} K_{i}\right)=\widetilde{H}^{n}\left(K_{i} / \partial_{Z} K_{i}\right) .
$$

Note that $K_{i} \subset \overline{B(0, i)}$ and $\partial_{Z} K_{i} \subset \partial \overline{B(0, i)}$, so $K_{i} / \partial_{Z} K_{i} \subset \overline{B(0, i)} / \partial \overline{B(0, i)}$. Thus, if $K_{i} \neq B(0, i)$, then $K_{i} / \partial_{Z} K_{i} \subset S^{n} \backslash\{*\}$. That is, $K_{i} / \partial_{Z} K_{i}$ is a compact set in $\mathbb{R}^{n}$, and $\widetilde{H}^{n}\left(K_{i} / \partial_{Z} K_{i}\right)=0$. Thus, if $Z=\mathbb{R}^{n}$, we have $H_{c}^{n}(Z)=\mathbb{Z}$. Otherwise, $H_{c}^{n}(Z)=0$.

Compactly supported cohomology is not invariant under quasi-isometries or uniform embeddings. The remainder of this section is distilled from [14, where compactly supported cohomology is generalized to a theory invariant under uniform embeddings. For our purposes, the basic ideas of this theory, made explicit below, are sufficient.

Definition 3.3. Let $X$ be a simplicial complex with the standard metric assigning each edge length 1 . Recall that a chain in $X$ is a formal linear combination of simplices. The support of a chain is the union of the simplices that have non-zero coefficients in the chain. The diameter of a chain is the diameter of its support. 
An acyclic metric simplicial complex $X$ is $k$-uniformly acyclic if there exists a function $\alpha$ such that any closed chain with diameter $d$ is the boundary of a $k+1$ chain of diameter at most $\alpha(d)$. If $X$ is $k$-uniformly acyclic for all $k$, we say that it is uniformly acyclic.

Likewise, we say that a metric simplicial complex $X$ is $k$-uniformly contractible if there exists a function $\alpha$ such that every continuous map $S^{k} \rightarrow X$ with image having diameter $d$ extends to a map $B^{k+1} \rightarrow X$ with diameter at most $\alpha(d)$. If $X$ is $k$-uniformly contractible for all $k$, we say it is uniformly contractible.

Remark 3.4. Rank one symmetric spaces and nilpotent Lie groups (with leftinvariant Riemannian metrics) are uniformly contractible and uniformly acyclic.

Lemma 3.5. Let $X, Y$ be uniformly contractible and geometrically finite metric simplicial complexes and $f: X \rightarrow Y$ a uniform embedding. Then there exists an iterated barycentric subdivision of $X$ and $R>0$ depending only on the uniformity constants of $f, X$, and $Y$ such that $f$ is approximated by a continuous simplicial map with additive error of at most $R$.

Proof. We first approximate $f$ by a continuous (but not simplicial) map by working on the skeleta of $X$. Starting with the 0 -skeleton, adjust the image of each vertex by distance at most 1 so that the image of each vertex of $X$ is a vertex of $Y$. Next, assuming inductively that $f$ is continuous on each $k$-simplex of $X$, we now extend to the $k+1$ skeleton using the uniform contractibility of $Y$. Since error was bounded on the $k$-simplices, it remains bounded on the $k+1$-skeleton.

Now that $f$ has been approximated by a continous map, a standard simplicial approximation theorem replaces $f$ by a continuous simplicial map, with bounded error depending only on the geometry of $X$ and $Y$ (see for example the proof of Theorem 2C.1 of [13]).

Lemma 3.6. Let $X$ and $Y$ be uniformly acyclic simplicial complexes and $f: X \rightarrow$ $Y$ a uniform embedding. Suppose furthermore that $f$ is a continuous simplicial map. Then if $H_{c}^{n}(X) \cong H_{c}^{n}(f X)$.

Proof. We first construct a left inverse $\rho$ of the map $f_{*}: C_{*}(X) \rightarrow C_{*}(f X)$ induced by $f$ on the chain complex of $X$, up to a chain homotopy $P$. That is, $P$ will be a map $C_{*}(X) \rightarrow C_{*+1}(X)$ satisfying, for each $c \in C_{*}(X)$, the homotopy condition

$$
\partial P c=c-\rho f_{*} c-P \partial c
$$

and furthermore with diameter of $P c$ controlled uniformly by the diameter of $c$.

We start with the 0 -skeleton. Each vertex $v^{\prime} \in f X$ is the image of some vertex $v \in X$ (not necessarily unique). Set $\rho\left(v^{\prime}\right)=v$, and extend by linearity to $\rho$ : $C_{0}(f X) \rightarrow C_{0}(X)$. To define $P$, let $v$ be an arbitrary vertex in $X$ and note that $\partial v=0$. We have to satisfy $\partial P v=v-\rho f_{*} v$. Since $X$ is acyclic, there exists a 1chain $P v$ satisfying this condition. Furthermore, note that $\rho f_{*} v$ is, by construction, a vertex such that $f\left(\rho f_{*} v\right)=f(v)$. Since $f$ is a uniform embedding, $d\left(\rho f_{*} v, v\right)$ is uniformly bounded above. Thus, $P v$ may be chosen using uniform acyclicity so that its diameter is also uniformly bounded above.

Assume next that $\rho$ and $P$ are defined for all $i<k$ with uniform control on diameters. Let $\sigma$ be a $k$-simplex in $X$. Then $\partial \rho f_{*} \sigma$ is a chain in $X$ whose diameter is bounded independently of $\sigma$. Then, by uniform acyclicity there is a chain $\sigma^{\prime}$ with $\partial \sigma^{\prime}=\partial \rho f_{*} \sigma$. We define $\rho(\sigma)=\sigma^{\prime}$. As before, we need to link $\sigma^{\prime}$ back to $\sigma$. We 
have

$$
\partial\left(\sigma-\sigma^{\prime}-P \partial \sigma\right)=\partial \sigma-\rho f_{*} \partial \sigma-\partial P \partial \sigma .
$$

By the homotopy condition (3.2), we further have

$$
\partial\left(\sigma-\sigma^{\prime}-P \partial \sigma\right)=\partial \sigma-\rho f_{*} \partial \sigma-\left(\partial \sigma-\rho f_{*} \partial \sigma-P \partial \partial \sigma\right)=0 .
$$

Thus, by bounded acyclicity there is a $k+1$ chain $P \sigma$ such that

$$
\partial P \sigma=\sigma-\sigma^{\prime}-P \partial \sigma
$$

as desired. We extend both $\rho$ and $P$ by linearity to all of $C_{k}(f X)$ and $C_{k}(X)$, respectively.

To conclude the argument, let $K$ be a compact subcomplex of $X$ and consider the complex $X /(X \backslash K)=K / \partial K$. The maps $P$ and $\rho \circ f_{*}$ on $C_{*}(X)$ induce maps on $C_{*}(K / \partial K)$, and the condition $\partial P c+P \partial c=c-\rho f_{*} c$ remains true for the induced maps and chains.

Because chain-homotopic maps on $C_{*}$ induce the same maps on homology, we have, for $h \in H_{*}(K / \partial K), h=\rho f_{*} h$. Conversely, $f_{*} \rho$ is the identity on cell complexes, so still the identity on homology. Thus, $H_{*}(K / \partial K) \cong H_{*}(f K / \partial f K)$. By duality, $H^{*}(f K / \partial f K) \cong H^{*}(K / \partial K)$.

Taking $K_{i}$ to be an exhaustion of $X$ by compact subcomplexes and taking a direct limit, we conclude that $H_{c}^{*}(X) \cong H_{c}^{*}(f X)$.

Corollary 3.7. Let $X$ and $Y$ be uniformly acyclic simplicial complexes and $f$ : $X \rightarrow Y$ a uniform embedding. There exists an $R>0$ depending only on the uniformity constants of $f, X$, and $Y$ so that $H_{c}^{n}\left(N_{R}(f X)\right) \cong H_{c}^{n}(X)$.

Proof. Lemma 3.5 approximates $f$ by a continuous simplicial map, within uniform additive error. Lemma 3.6 shows that the resulting approximation induces an isomorphism on compactly supported cohomology.

Theorem 3.8 (Coarse co-Hopficity). Let $\left(X, d_{X}\right)$ be a manifold homeomorphic to $\mathbb{R}^{n}$, with $d_{X}$ a path metric that is uniformly acyclic and uniformly contractible. For each pair of non-decreasing functions $\alpha, \omega:[0, \infty) \rightarrow \mathbb{R}$ with $\alpha(t)<\omega(t)$ and $\lim _{t \rightarrow \infty} \alpha(t)=\infty$, there exists a $C^{\prime}$ such that any $(\alpha, \omega)$-coarse embedding $f: X \rightarrow X$ is $C^{\prime}$-coarsely surjective.

Proof. By Corollary 3.7 there is a uniform $R>0$ such that $H_{c}^{n}\left(N_{R}(f X)\right) \cong$ $H_{c}^{n}(X) \cong \mathbb{Z}$. By Lemma [3.2. $N_{R}(f X)=X$. Taking $C^{\prime}=C+2 R$ completes the proof.

\section{MAin RESUlT}

Theorem 4.1 (Quasi-Isometric co-Hopficity). Let $\Omega \subset X$ and $\Omega^{\prime} \subset X^{\prime}$ be equivariant truncated spaces and $f:\left(\Omega, d_{\Omega}\right) \rightarrow\left(\Omega^{\prime}, d_{\Omega^{\prime}}\right)$ a quasi-isometric embedding. Then $f$ is coarsely surjective with respect to the truncated metric $d_{\Omega}$.

Proof. By Lemma 2.10, we may assume that $f$ maps boundary horospheres of $\Omega$ to boundary horospheres of $\Omega^{\prime}$. By Theorem 3.8, $f$ is a surjection up to a constant independent of the boundary horosphere in question. We then have an extension $F: X \rightarrow X^{\prime}$, as in Definition 2.11 
By Lemma 2.12 for each boundary horoball $\mathcal{B}$, the restriction $\left.F\right|_{\mathcal{B}}$ is a quasiisometry. By assumption, $\left.F\right|_{\Omega}$ is a $d_{\Omega}$-quasi-isometry, so $\left.F\right|_{\Omega}$ is a $d$-uniform embedding by Proposition 2.8. Since $X$ is a path metric space, $F$ is then coarsely Lipschitz on all of $X$.

We now show that $F$ is a uniform embedding by establishing a lower bound for distances between image points. Recall that all distances are measured with respect to $d=d_{X}$ unless another metric is explicitly mentioned.

Let $L \gg 2$ so that $F$ is coarsely $L$-Lipschitz and $\left.F\right|_{\mathcal{B}}$ is coarsely $L$-co-Lipschitz for every boundary horoball $\mathcal{B}$. Let $\alpha, \omega$ be increasing proper functions so that $f$ is an $(\alpha, \omega)$-uniform embedding.

Let $x_{1}, x_{2} \in X$ with $d\left(x_{1}, x_{2}\right) \gg 0$. We need to provide a lower bound for $d\left(F x_{1}, F x_{2}\right)$ in terms of $d\left(x_{1}, x_{2}\right)$. Clearly, the lower bound will go to $\infty$ since $F$ is an isometry along vertical geodesics in horoballs. There are four cases to consider; in all cases we can ignore additive noise by working with sufficiently large $d\left(x_{1}, x_{2}\right)$ and slightly increasing $L$.

(1) Let $x_{1}, x_{2} \in \mathcal{B}$ for the same horoball $\mathcal{B}$. Then $d\left(f x_{1}, f x_{2}\right)>d\left(x_{1}, f x_{2}\right) / L$.

(2) Let $x_{1}, x_{2} \in \Omega$. This case is controlled by the uniform embeddings $\Omega \hookrightarrow X$ and $\Omega^{\prime} \hookrightarrow X^{\prime}$ (Proposition 2.8) and the $d_{\Omega^{-}}$quasi-isometry constants of $f$.

(3) Let $x_{1} \in \Omega, x_{2} \in \mathcal{B}$ for a horoball $\mathcal{B}$. Let $b \in B$ be the closest point to $x_{1}$. Then by Lemma 2.2. $\left[x_{1}, b\right] \cup\left[b, x_{2}\right]$ is a $C$-quasi-geodesic for a universal $C$ depending only on $X$ and $X^{\prime}$ (see also Figure 1). We consider two sub-cases:

Suppose that $d\left(x_{1}, b\right)>d\left(x_{1}, x_{2}\right) / L^{3}$. Let $b^{\prime} \in f \mathcal{B}$ be the closest point to $f x_{1}$. Then by definition of $b$, we have

$$
d\left(f^{-1} b^{\prime}, x_{1}\right) \geq d\left(b, x_{1}\right) \geq d\left(x_{1}, x_{2}\right) / L^{3} .
$$

Using Lemma 2.4 we conclude

$$
d\left(f x_{1}, f x_{2}\right) \geq d\left(b^{\prime}, f x_{2}\right) \geq \alpha\left(d\left(f^{-1}, x_{2}\right)\right) \geq \alpha\left(d\left(x_{1}, x_{2}\right) / L^{3}\right) .
$$

Suppose, instead, that $d\left(x_{1}, b\right) \leq d\left(x_{1}, x_{2}\right) / L^{3}$. Then we have the estimate $d\left(f x_{1}, f b\right) \leq d\left(x_{1}, x_{2}\right) / L^{2}$. We also have $d\left(x_{2}, b\right) \approx d\left(x_{1}, x_{2}\right)$, so $d\left(f x_{2}, f b\right) \geq d\left(x_{1}, x_{2}\right) / L$. Consider now $b^{\prime} \in \mathcal{B}$, the closest point to $f x_{1}$. By Lemma 2.2, $d\left(f b, f b^{\prime}\right) \leq d\left(f b, f x_{1}\right)$. Thus,

$$
d\left(f x_{1}, f x_{2}\right) \geq d\left(x_{1}, x_{2}\right) / L-d\left(x_{1}, x_{2}\right) / L^{2}
$$

(4) Let $x_{1} \in \mathcal{B}_{1}, x_{2} \in \mathcal{B}_{2}$ be in disjoint horoballs. This case is identical to the previous one, except one uses Lemma 2.3 rather than Lemma 2.2 .

We have then provided a lower bound for $d\left(F x_{1}, F x_{2}\right)$ for any pair of points $x_{1}, x_{2} \in X$. Thus, the extended map $F$ is a coarse embedding. By Theorem 3.8 . $F$ is then coarsely surjective. Namely, there exists $R>0$ so that $N_{R}(F(X))=X^{\prime}$ (the neighborhood is taken with respect to $d$ ).

We now show that the coarse surjectivity of $F$ with respect to $d$ implies the coarse surjectivity of $f$ with respect to $d_{\Omega}$. 
Let $\omega^{\prime} \in \Omega^{\prime}$ be an arbitrary point. Since $F$ is coarsely surjective, there exists $x \in X$ so that $d_{X^{\prime}}\left(f(x), \omega^{\prime}\right) \leq R$. If $x \in \Omega$, then we have shown that $\omega^{\prime} \in N_{R}(f(\Omega))$. Otherwise, $x$ is contained in a horoball associated with $\Omega$. In appropriate horospherical coordinates, the horoball is given by $S \times\left(t_{0}, \infty\right)$ and $x$ can be written as $\left(s_{1}, t_{1}\right)$, with $t_{1}>t_{0}$. Likewise, $f(x)$ has coordinates $\left(s_{1}^{\prime}, t_{1}^{\prime}\right)$, with $\left(t_{1}^{\prime}>t_{0}^{\prime}\right)$. Furthermore, we have $f\left(s_{1}, t_{0}\right)=\left(s_{1}^{\prime}, t_{0}\right)$. Now, $\omega^{\prime} \in \Omega^{\prime}$, so it has horospherical coordinates $\left(s_{2}^{\prime}, t_{2}^{\prime}\right)$ with $t_{2}^{\prime}<t_{0}^{\prime}$. It is easy to see that

$$
\begin{aligned}
R & \geq d_{X^{\prime}}\left(\omega^{\prime},\left(s_{1}^{\prime}, t_{1}^{\prime}\right)\right) \geq d_{X^{\prime}}\left(\omega^{\prime},\left(s_{1}^{\prime}, t_{0}^{\prime}\right)\right) \\
& =d_{X^{\prime}}\left(\omega^{\prime}, f\left(s_{1}, t_{0}\right)\right) \geq d_{X^{\prime}}\left(\omega^{\prime}, f(\Omega)\right) .
\end{aligned}
$$

Thus, for an arbitrary $\omega^{\prime} \in \Omega^{\prime}$ we have $d_{X^{\prime}}\left(\omega^{\prime}, f(\Omega)\right) \leq R$. Because $\Omega^{\prime} \hookrightarrow X^{\prime}$ is a uniform embedding, this implies that $f: \Omega \rightarrow \Omega^{\prime}$ is coarsely surjective.

\section{ACKNOWLEDGEMENT}

The authors would like to thank Misha Kapovich for useful conversations.

\section{REFERENCES}

1. I. Belegradek, On co-Hopfian nilpotent groups, Bull. London Math. Soc. 35 (2003), no. 6, 805-811. MR2000027 (2004i:20060)

2. I. Belegradek and A. Szczepański, Endomorphisms of relatively hyperbolic groups, Internat. J. Algebra Comput. 18 (2008), no. 1, 97-110, with an appendix by Oleg V. Belegradek. MR2394723 (2009a:20069)

3. M. Bonk, and O. Schramm, Embeddings of Gromov hyperbolic spaces. Geom. Funct. Anal. 10 (2000), no. 2, 266-306. MR1771428 (2001g:53077)

4. H. Bourdon, and M. Pajot, Poincaré inequalities and quasiconformal structure on the boundary of some hyperbolic buildings, Proc. Amer. Math. Soc. 127 (1999), no. 8, 2315-2324. MR.1610912 (99j:30024)

5. M. Bourdon, Immeubles hyperboliques, dimension conforme et rigidité de Mostow, Geom. Funct. Anal. 7 (1997), no. 2, 245-268. MR1445387 (98c:20056)

6. M. Bridson and A. Haefliger, Metric spaces of non-positive curvature, Grundlehren der Mathematischen Wissenschaften [Fundamental Principles of Mathematical Sciences], vol. 319, Springer-Verlag, Berlin, 1999. MR 1744486 (2000k:53038)

7. M. R. Bridson, D. Groves, J. A. Hillman, and G. J. Martin, Confinitely Hopfian groups, open mappings and knot complements. Groups Geom. Dyn. 4 (2010), no. 4, 693-707. MR2727659 (2011j:20103)

8. P. de la Harpe, Topics in geometric group theory, Chicago Lectures in Mathematics, University of Chicago Press, Chicago, IL, 2000. MR1786869 (2001i:20081)

9. T. Delzant and L. Potyagailo, Endomorphisms of Kleinian groups, Geom. Funct. Anal. 13 (2003), no. 2, 396-436. MR1982149 (2004c:20087)

10. D. Epstein, J. Cannon, D. Holt, S. Levy, M. Paterson, and W. Thurston, Word processing in groups, Jones and Bartlett Publishers, Boston, MA, 1992. MR 1161694 (93i:20036)

11. A. Eskin, Quasi-isometric rigidity of nonuniform lattices in higher rank symmetric spaces, J. Amer. Math. Soc. 11 (1998), no. 2, 321-361. MR.1475886 (98g:22005)

12. B. Farb and L. Mosher, A rigidity theorem for the solvable Baumslag-Solitar groups, Invent. Math. 131 (1998), no. 2, 419-451, with an appendix by Daryl Cooper. MR 1608595 (99b:57003)

13. A. Hatcher, Algebraic topology, Cambridge University Press, Cambridge, 2002. MR 1867354 (2002k:55001)

14. M. Kapovich and B. Kleiner, Coarse Alexander duality and duality groups, J. Differential Geom. 69 (2005), no. 2, 279-352. MR2168506 (2007c:57033)

15. S. Merenkov, A Sierpiński carpet with the co-Hopfian property, Invent. Math. 180 (2010), no. 2, 361-388. MR2609245 (2011c:30141)

16. R. E. Schwartz, The quasi-isometry classification of rank one lattices, Inst. Hautes Études Sci. Publ. Math. (1995), no. 82, 133-168 (1996). MR.1383215(97c:22014) 
17. Z. Sela, Structure and rigidity in (Gromov) hyperbolic groups and discrete groups in rank 1 Lie groups. II, Geom. Funct. Anal. 7 (1997), no. 3, 561-593. MR1466338 (98j:20044)

18. Z. Sela, Endomorphisms of hyperbolic groups. I. The Hopf property. Topology 38 (1999), no. 2, 301-321. MR:1660337 (99m:20081)

Department of Mathematics, University of Illinois at Urbana-Champaign, 1409 West Green Street, Urbana, Illinois 61801

URL: http://www.math.uiuc.edu/ kapovich/

E-mail address: kapovich@math.uiuc.edu

Department of Mathematics, University of Illinois at Urbana-Champaign, 1409 West Green Street, Urbana, Illinois 61801

$U R L:$ http://lukyanenko.net

E-mail address: anton@lukyanenko.net 